\title{
Statistical properties of atmospheric greenhouse gas measurements: Looking down from space and looking up from the ground
}

\author{
Bohai Zhang ${ }^{\mathrm{a}}$, Noel Cressie ${ }^{\mathrm{a}}$, Debra Wunch ${ }^{\mathrm{b}}$ \\ a National Institute for Applied Statistics Research Australia, University of \\ Wollongong, NSW, 2522, Australia \\ b Department of Physics and School of the Environment, University of Toronto, \\ Toronto, ON, M5S 3E8, Canada
}

\begin{abstract}
Satellite remote sensing platforms can collect measurements on a global scale within a few days, which provides an unprecedented opportunity to characterize and understand the spatio-temporal variability of environmental variables. Because of the additional challenges of making precise and accurate measurements from space, it is essential to validate satellite remote sensing datasets with highly precise and accurate ground-based measurements. The focus of this article is on two sets of measurements: Atmospheric column-averaged carbon dioxide $\left(\mathrm{CO}_{2}\right)$ collected by the Orbiting Carbon Observatory-2 (OCO-2) mission in its target mode of operation; and ground-based data used for validation from the Total Carbon Column Observing Network (TCCON). The current statistical modeling of the relationship between the less-precise OCO-2 satellite data $(Y)$ and the more-precise TCCON ground-based data $(X)$ assumes a linear regression and heteroscedastic measurement errors that reside in both the OCO-2 data and the TCCON data. To obtain consistent estimates of the regression coefficients, it is critical to determine the error variance of each datum in the regression. In this article, a rigorous statistical procedure is presented for obtaining these error variances through modeling the spatial and/or temporal dependence structure in the OCO-2 and TCCON datasets. Numerical results for analyzing data at the Lamont TCCON station and the corresponding OCO-2 target-mode data (orbit number 3590) illustrate our procedure.
\end{abstract}

Key words: atmospheric carbon dioxide, errors in variables, OCO-2, spatial statistics, TCCON, time series 


\section{Introduction}

Satellite remote sensing measurements of Earth's surface and atmosphere provide global coverage within a matter of days. This helps scientists understand the spatio-temporal distribution of environmental processes. Examples of remote sensing datasets of this type include measurements of atmospheric trace gases (e.g., carbon dioxide, methane, ozone), sea surface temperature, sea-ice extent, solar-induced fluorescence from plants, aerosols, and so forth. These remote sensing measurements from space require validation from well characterized ground-based measurements to ensure their accuracy and precision throughout the satellite's mission. This is often achieved through fitting a linear regression relationship between coincident ground-based and satellitebased measurements, where uncertainties are present in both the dependent $(Y)$ and independent $(X)$ variables. It is usually not appropriate to assume error variances are homogeneous, and hence correctly determining the error variance of each $X$-value and $Y$-value in the regression is of critical importance.

In this article, we estimate the error variances of these values by incorporating spatial dependence for the satellite-based measurements and temporal dependence for the ground-based measurements. Finding these variances is key to accurately estimating the regression parameters. The problem is generally relevant to many topics in chemistry, physics, and the biogeosciences; in this article, we focus on the Orbiting Carbon Observatory-2 (OCO-2) validation program [1]. The OCO-2 instrument measures carbon dioxide $\left(\mathrm{CO}_{2}\right)$ in Earth's atmosphere.

The OCO-2 mission aims to provide the atmospheric measurements required to understand better the carbon cycle, which is the cycling of carbon (mostly in the form of $\mathrm{CO}_{2}$ in the atmosphere) between the oceans, land, terrestrial biosphere, and atmosphere. The main sinks of $\mathrm{CO}_{2}$ are the oceans, which dissolve $\mathrm{CO}_{2}$ into seawater to form carbonic acid, and the terrestrial biosphere, in which plants, through photosynthesis, convert $\mathrm{CO}_{2}$ into the sugars necessary to grow [2]. There are many sources of atmospheric $\mathrm{CO}_{2}$, primarily fossil-fuel burning (e.g., coal, petroleum, natural gas), which oxidizes carboncontaining fuels to produce $\mathrm{CO}_{2}$; and land use, which both alters the surface albedo and on average reduces the land $\mathrm{CO}_{2}$ sink [2]. Other significant sources of $\mathrm{CO}_{2}$ include industrial processes such as cement production, where limestone $\left(\mathrm{CaCO}_{3}\right)$ is chemically converted into calcium oxide $(\mathrm{CaO})$ and $\mathrm{CO}_{2}$ is produced as a by-product $[2,3]$.

Due to human activities, $\mathrm{CO}_{2}$ concentrations in Earth's atmosphere have been increasing: The atmospheric $\mathrm{CO}_{2}$ concentration has increased from about 280 parts per million (ppm) since the beginning of the industrial revolution in the 
1700 s to about 400 ppm today. The percentage of each year's $\mathrm{CO}_{2}$ emissions that remains in the atmosphere has also been increasing in the past 50 years. According to [4], there is evidence that from 1959 to 2008, the fraction of $\mathrm{CO}_{2}$ emissions that remains in the atmosphere each year is likely to have increased from $40 \%$ to $45 \%$. The increasing levels of $\mathrm{CO}_{2}$ and other greenhouse gases in the atmosphere are the primary drivers of Earth's surface temperature increases.

The goals of the OCO-2 mission are to measure $\mathrm{CO}_{2}$ with high enough precision and accuracy to distinguish between the sources and sinks of $\mathrm{CO}_{2}$ on regional scales, and to quantify the seasonal, latitudinal, and interannual variability of $\mathrm{CO}_{2}$ [5]. To achieve this goal, measurement precision and accuracy must be better than one part per million (ppm) of $\mathrm{CO}_{2}$ (i.e., 0.25\%) [6]. This is a difficult task and, thus, the method to ensure that the OCO-2 data are sufficiently accurate is critical. The standard $\mathrm{CO}_{2}$ gas scale is set by the World Meteorological Organization (WMO); to tie the OCO-2 data to that standard scale requires a so-called transfer standard between the WMO-calibrated instruments and the remote sensing OCO-2 measurements. The Total Carbon Column Observing Network (TCCON, [7]) acts as this transfer standard, since TCCON is tied to the WMO scale through comparisons with WMO-traceable aircraft and balloon-borne measurements [8]. There are over 20 ground-based atmospheric observing stations in the TCCON, which are at fixed locations throughout the world.

To compare OCO-2 data with TCCON data, a special observation mode was designed for the OCO-2 satellite, called "target mode." In this mode, the OCO-2 spacecraft turns to "stare" at a ground location (typically a TCCON station) as it passes overhead, recording thousands of measurements in a small geographic area $(\sim 0.2 \times 0.2$ degrees $)$ over just a few minutes $(\sim 5$ minutes $)$. Under these conditions, changes in atmospheric $\mathrm{CO}_{2}$ abundances are negligibly small, and the OCO-2 data obtained from looking down from space are directly coincident and comparable with the TCCON data obtained from looking up from the ground [9]. Currently, the OCO-2 target-mode maneuver occurs at 21 TCCON locations and at other locations as needed [9].

For each of these target-mode maneuvers, thousands of individual OCO-2 observations and their coincident 60 or so individual TCCON observations are aggregated over space and time, respectively, to form one $(X, Y)$ point in a regression analysis. After some preprocessing of the OCO-2 data, a linear regression between the aggregated OCO-2 data $(Y)$ and the aggregated TCCON data $(X)$ is fitted, and the deviation of the OCO-2 $\mathrm{CO}_{2}$ product from the WMO scale is quantified [10]. This deviation is removed from the OCO-2 data, using the fitted regression line, before its use in scientific studies. Thus, fitting this regression line correctly is imperative, and we show in this article how important it is to know or estimate the error variance of each OCO-2 
value and each TCCON value used in the regression.

The result we obtain for the variance of the OCO-2 value in the regression can be used in contexts that go beyond this calibration study. For example, flux inversions usually work with spatially aggregated mole-fraction data (e.g., from OCO-2 retrievals), and our research demonstrates how the spatial covariance of the mole-fraction field determines the all-important variances of aggregates.

Another benefit of our research is for small area analysis [10-12], where we are able to account for dependence in the individual measurements that are aggregated to obtain an OCO-2 value (spatial aggregation) or a TCCON value (temporal aggregation). This statistical dependence results in a modification (often reduction) of the number of independent observations, to a number that we call the effective sample size. The interpretation of these, in terms of reduced information content in the small areas, is powerful and intuitive.

The rest of the paper is organized as follows. In Section 2, we provide the details of the errors-in-variables model that is currently used by the OCO-2 validation team. In Section 3, we discuss the selection of weights in the regression of OCO-2 on TCCON, and we provide sufficient conditions for obtaining unbiased estimating equations of regression parameters. In this section, we also illustrate, through simulation, the benefits of using an unbiased estimating equation for the regression slope $b$. We elaborate the statistical-analysis procedures for individual TCCON and OCO-2 data in Section 4, focusing on modeling the temporal and spatial data-dependence structures of the TCCON and OCO-2 data, respectively. In Section 5, we provide the formulas for computing the variances of the values fitted in the regression, and we use the datasets from the TCCON site at Lamont combined with the OCO-2 orbit number 3590 as an example. Concluding remarks are given in Section 6, and the paper finishes with a technical appendix.

\section{The errors-in-variables model used for OCO-2 calibration}

Version 7 of the OCO-2 data product is publicly available and can be found at [13]. The regression procedure used to obtain version 7 is described in [10], as follows. Let $\left(X_{i}, Y_{i}\right)$ be a pair of TCCON and OCO-2 target-mode observations, where $i$ indexes a combination of TCCON site and OCO-2 orbit number. Suppose there are $i=1, \ldots, N$ such combinations. The errors-invariables model in $[14,15]$ was used to model the linear relationship between these pairs, with TCCON as the independent variable $(X)$ and OCO-2 as the dependent variable $(Y)$. This model also has important applications in chemistry (e.g., see [16-18]). An iterative optimization algorithm in [15] was used to estimate the regression coefficients in the case of OCO-2 regressed on 


\section{TCCON.}

In this article, we show that the least-sum-of-weighted-squares estimators of the regression coefficients in $[14,15]$ can be viewed as maximum (profile) likelihood estimators under Gaussian-error assumptions. Second, we show that the estimators are (asymptotically) unbiased and (statistically) efficient when the regression weights are properly specified as being inversely proportional to $\operatorname{var}\left(X_{i}\right)$ and $\operatorname{var}\left(Y_{i}\right)$, respectively, for $i=1, \ldots, N$. Third, we show that misspecified weights result in biased estimating equations of the regression parameters, and hence the resulting regression-parameter estimates and regression line can be biased (Section 3). Last, we show that TCCON datasets are weakly correlated in time and OCO-2 datasets are highly correlated in space, which must be accounted for when estimating the variances of $X_{i}$ and $Y_{i}$, respectively (Section 4).

Generally, suppose that $\left\{\left(X_{1}, Y_{1}\right),\left(X_{2}, Y_{2}\right), \ldots,\left(X_{N}, Y_{N}\right)\right\}$ are $N$ pairs of groundmonitoring-station data $(X)$ paired with satellite remote sensing data $(Y)$. Since the data collected by both the satellite and the ground-monitoring stations have measurement errors associated with them, an errors-in-variables model is appropriate for modeling their relationship. Assume that $E\left(X_{i}\right)=x_{i}$ and $E\left(Y_{i}\right)=y_{i}$, where $x_{i}$ and $y_{i}$ are (fixed but unknown) true values of $X_{i}$ and $Y_{i}$, respectively. Because both datasets attempt to measure the same variable (e.g., in our application, column-averaged $\mathrm{CO}_{2}$ ), it is expected that there is a strong relationship between them. Fitting a linear relationship provides a straightforward way for correcting the bias in the satellite data using the more accurate data from the ground-monitoring stations.

In [10], the current errors-in-variables model for producing version 7 of the OCO-2 data product is given as follows. For $i=1, \ldots, N$,

$$
\begin{aligned}
& X_{i}=x_{i}+\epsilon_{x, i}, \\
& Y_{i}=y_{i}+\epsilon_{y, i}, \\
& y_{i}=a+b x_{i},
\end{aligned}
$$

where the measurement-error terms, $\epsilon_{x, i}$ and $\epsilon_{y, i}$, are assumed to have mean zero and variances $\tilde{\sigma}_{x, i}^{2}$ and $\tilde{\sigma}_{y, i}^{2}$, respectively. It is also reasonable to assume that they are mutually independent for all $i=1, \ldots, N$. The model (1) is also known as a functional model (e.g., see $[19,20])$.

It is important to clarify that $X_{i}$ and $Y_{i}$ are aggregated data calculated from a set of individual TCCON observations and a set of individual OCO-2 targetmode observations, respectively. Let $n_{x, i}$ and $n_{y, i}$ denote sample sizes of individual TCCON and OCO-2 observations for obtaining $X_{i}$ and $Y_{i}$, respectively. In this article, we derive the effective sample sizes $\tilde{n}_{x, i}$ and $\tilde{n}_{y, i}$ in 


$$
\tilde{\sigma}_{x, i}^{2} \equiv \operatorname{var}\left(X_{i}\right)=\sigma_{x, i}^{2} / \tilde{n}_{x, i}, \quad \tilde{\sigma}_{y, i}^{2} \equiv \operatorname{var}\left(Y_{i}\right)=\sigma_{y, i}^{2} / \tilde{n}_{y, i},
$$

where $\sigma_{x, i}^{2}$ and $\sigma_{y, i}^{2}$ are variances of a single TCCON observation and a single OCO-2 observation, respectively. Recall that $\tilde{n}_{x, i}$ and $\tilde{n}_{y, i}$ are the effective sample sizes that account for dependence in the observations, and they are generally different from their respective $n_{x, i}$ and $n_{y, i}$. Strong positive correlations between individual observations results in effective sample sizes much smaller than actual sample sizes (Section 5).

A least-sum-of-weighted-squares criterion was proposed in [21]: This leads to estimating $a$ and $b$ by minimizing

$$
S(a, b)=\sum_{i=1}^{N} \frac{w_{x, i} w_{y, i}}{b^{2} w_{y, i}+w_{x, i}}\left(Y_{i}-a-b X_{i}\right)^{2}
$$

with respect to $a$ and $b$. The resulting estimates, $\hat{a}_{l w s}$ and $\hat{b}_{l w s}$, are functions only of the data $\left\{\left(X_{i}, Y_{i}\right)\right\}_{i=1}^{N}$, for given regression weights $\left\{w_{x, i}\right\}_{i=1}^{N}$ and $\left\{w_{y, i}\right\}_{i=1}^{N}$. The regression weights $w_{x, i}$ and $w_{y, i}$ are pre-specified, and they should be chosen to be the inverse of the variances of $X_{i}$ and $Y_{i}$, respectively.

Least-sum-of-weighted-squares estimators are, under certain assumptions, maximum likelihood estimators. In [22], it can be seen that the sum-of-weightedsquares function in (3) is equivalent to the profile log-likelihood function, for independent Gaussian measurement errors $\epsilon_{x, i}$ and $\epsilon_{y, i}$ in the model (1) and weights specified as $w_{x, i}=1 / \tilde{\sigma}_{x, i}^{2}$ and $w_{y, i}=1 / \tilde{\sigma}_{y, i}^{2} ; i=1, \ldots, N$. Therefore, the least-sum-of-weighted-squares estimators, $\hat{a}_{l w s}$ and $\hat{b}_{l w s}$, are also maximum (profile) likelihood estimators, when the weights are appropriately chosen.

Partially differentiating (3) with respect to $a$ and $b$, one can obtain (see [21]),

$$
b=\frac{\sum_{i=1}^{N} Z_{i}^{2}\left(Y_{i}-\bar{Y}_{w}\right)\left(\frac{X_{i}-\bar{X}_{w}}{w_{y, i}}+\frac{b\left(Y_{i}-\bar{Y}_{w}\right)}{w_{x, i}}\right)}{\sum_{i=1}^{N} Z_{i}^{2}\left(X_{i}-\bar{X}_{w}\right)\left(\frac{X_{i}-\bar{X}_{w}}{w_{y, i}}+\frac{b\left(Y_{i}-\bar{Y}_{w}\right)}{w_{x, i}}\right)},
$$

where $Z_{i} \equiv\left(w_{x, i} w_{y, i}\right) /\left(b^{2} w_{y, i}+w_{x, i}\right)$ depends on $b, \bar{X}_{w}=\sum_{i} Z_{i} X_{i} / \sum_{i} Z_{i}$, and $\bar{Y}_{w}=\sum_{i} Z_{i} Y_{i} / \sum_{i} Z_{i}$. Based on equation (4), [15] proposed an algorithm that solves for $b$ iteratively. If it converges, the resulting estimate is $\hat{b}_{l w s}$. Then the corresponding estimate of $a$ is $\hat{a}_{l w s}=\bar{Y}_{w}-\hat{b}_{l w s} \bar{X}_{w}$, where $\bar{X}_{w}$ and $\bar{Y}_{w}$ are evaluated at $b=\hat{b}_{l w s}$. 


\section{Unbiased estimation of regression parameters}

In this section, we find sufficient conditions under which the least-sum-ofweighted-squares estimators, $\hat{a}_{l w s}$ and $\hat{b}_{l w s}$, are (asymptotically) unbiased. The estimating equations for regression parameters $a$ and $b$ are $\frac{\partial S(a, b)}{\partial a}=0$ and $\frac{\partial S(a, b)}{\partial b}=0$, respectively; the estimating equations are unbiased if $E\left(\frac{\partial S(a, b)}{\partial a}\right)=0$ and $E\left(\frac{\partial S(a, b)}{\partial b}\right)=0$, for all $a, b \in \mathbb{R}$. Under regularity conditions, unbiased estimating equations result in consistent (asymptotically unbiased) estimators $[23,24]$. Therefore, unbiasedness of estimating equations of $a$ and $b$ is a desirable property.

Recall that the weights $\left\{w_{x, i}\right\}$ and $\left\{w_{y, i}\right\}$ are pre-specified; then

$$
\frac{\partial S(a, b)}{\partial a}=\sum_{i=1}^{N} \frac{-2 w_{x, i} w_{y, i}}{b^{2} w_{y, i}+w_{x, i}}\left(Y_{i}-a-b X_{i}\right),
$$

and hence $E\left(\frac{\partial S(a, b)}{\partial a}\right)=0$. Further,

$$
\begin{gathered}
\frac{\partial S(a, b)}{\partial b}=\sum_{i=1}^{N} \frac{-2 w_{x, i} w_{y, i}}{\left(b^{2} w_{y, i}+w_{x, i}\right)^{2}}\left\{b\left(Y_{i}-a\right)^{2} w_{y, i}+X_{i}\left(Y_{i}-a\right) w_{x, i}\right. \\
\left.-b^{2} X_{i}\left(Y_{i}-a\right) w_{y, i}-b X_{i}^{2} w_{x, i}\right\}
\end{gathered}
$$

and hence

$$
E\left(\frac{\partial S(a, b)}{\partial b}\right)=\sum_{i=1}^{N} \frac{-2 b w_{x, i} w_{y, i}}{\left(b^{2} w_{y, i}+w_{x, i}\right)^{2}}\left(\tilde{\sigma}_{y, i}^{2} w_{y, i}-\tilde{\sigma}_{x, i}^{2} w_{x, i}\right)
$$

since $E\left(X_{i}^{2}\right)=\tilde{\sigma}_{x, i}^{2}+x_{i}^{2}, E\left(\left(Y_{i}-a\right)^{2}\right)=\tilde{\sigma}_{y, i}^{2}+b^{2} x_{i}^{2}$, and $E\left(X_{i}\left(Y_{i}-a\right)\right)=b x_{i}^{2}$. Recall that $\tilde{\sigma}_{x, i}^{2}=\operatorname{var}\left(X_{i}\right)$ and $\tilde{\sigma}_{y, i}^{2}=\operatorname{var}\left(Y_{i}\right)$.

From (5), if

$$
\frac{w_{x, i}}{w_{y, i}}=\frac{\tilde{\sigma}_{y, i}^{2}}{\tilde{\sigma}_{x, i}^{2}}
$$

then $E\left(\frac{\partial S(a, b)}{\partial b}\right)=0$, for all $a, b \in \mathbb{R}$. The solution to (5) is $\hat{b}_{l w s}$, which is consistent if (6) holds. That is, provided the ratio of the weights associated with $X_{i}$ and $Y_{i}$ is equal to the reciprocal of the ratio of their corresponding true variances, $\hat{b}_{l w s}$ is consistent. In the following subsection, we use a simulation example to show that when the regression weights are misspecified, the leastsum-of-weighted-squares estimator of $b$ is biased with a large mean squared 
error (MSE).

\subsection{Effects of regression weights on estimating the slope parameter $b$}

In this subsection, we use simulation to show the effect of misspecified weights on the least-sum-of-weighted-squares estimators of $a$ and $b$. The special case of the model (1) with $a=0$ will be featured, because that is the assumption made when the OCO-2 datasets are calibrated using the TCCON datasets. The intercept $a$ is fixed at zero to reflect that when the true TCCON value is zero, the corresponding OCO-2 value should also be zero. To provide a more general formulation, at the end of this subsection we discuss inference for the model given by (1) with both $a$ and $b$ to be estimated.

To illustrate the effect of the regression weights on estimating $b$, consider the following artificial example based on simulation, where the units of $X$ and the units of $Y$ are arbitrary and not related to our application to $\mathrm{CO}_{2}$ mole fraction. We first generated the true covariate values $\left\{x_{i}\right\}_{i=1}^{N}$ from a Gaussian distribution, $N\left(10,2^{2}\right)$, and we set the true response values $\left\{y_{i}\right\}_{i=1}^{N}$ to be given by: $y_{i}=0.8 x_{i}$. That is, the true value of $a$ is 0 and the true value of $b$ is 0.8 . Then $X_{i}$ was randomly generated from a Gaussian distribution with mean $x_{i}$ and variance $\tilde{\sigma}_{x, i}^{2}=0.5$, while $Y_{i}$ was randomly generated from a Gaussian distribution with mean $y_{i}$ and variance $\tilde{\sigma}_{y, i}^{2}=1.5$. This was repeated independently for $i=1, \ldots, N$. Thus, in the simulation, the true ratio of $\operatorname{var}\left(Y_{i}\right)$ to $\operatorname{var}\left(X_{i}\right)$ is $\tilde{\sigma}_{y, i}^{2} / \tilde{\sigma}_{x, i}^{2}=3$, for all $i=1, \ldots, N$.

We estimated $b$ under four different scenarios:

1) $w_{x, i}=1 / 0.5, w_{y, i}=1 / 1.5$, corresponding to the ideal case that specifies the weights of $X_{i}$ and $Y_{i}$ to be the reciprocals of their respective true variances ("True");

2) $w_{x, i}=1, w_{y, i}=1 / 3$, corresponding to misspecification of the weights but correct specification of their ratio ("Equal");

3) $w_{x, i}=1 / 2, w_{y, i}=2$, corresponding to a misspecification of the ratio, where $w_{x, i} / w_{y, i}=1 / 4$ is smaller than the correct ratio, $\tilde{\sigma}_{y, i}^{2} / \tilde{\sigma}_{x, i}^{2}=3$ ("Smaller");

4) $w_{x, i}=1, w_{y, i}=1 / 10$, corresponding to a misspecification of the ratio, where $w_{x, i} / w_{y, i}=10$ is bigger than the correct ratio, $\tilde{\sigma}_{y, i}^{2} / \tilde{\sigma}_{x, i}^{2}=3$ ("Bigger").

The slope parameter $b$ was estimated by minimizing the sum-of-weightedsquares objective function in (3). 
Table 1

Parameter estimation of the slope parameter $(b=0.8)$ under different specifications of weights. The rows "True," "Equal," "Smaller," and "Bigger" show the results for Scenarios 1-4, respectively. The $95 \%$ confidence interval is obtained as the sample mean plus/minus twice its standard error calculated from the simulation. The results are based on 200 simulated datasets for each of the four scenarios, and each of the three values of $N$.

\begin{tabular}{|l|cc|c|c|}
\hline \hline$N=150$ & Mean & Median & MSE & $95 \%$ CI \\
\hline True & 0.79945 & 0.79995 & $1.137 \cdot 10^{-4}$ & $(0.79794,0.80096)$ \\
Equal & 0.79945 & 0.79995 & $1.137 \cdot 10^{-4}$ & $(0.79794,0.80096)$ \\
Smaller & 0.81101 & 0.81218 & $2.368 \cdot 10^{-4}$ & $(0.80949,0.81254)$ \\
Bigger & 0.79701 & 0.79758 & $1.215 \cdot 10^{-4}$ & $(0.79550,0.79851)$ \\
\hline \hline$N=500$ & Mean & Median & MSE & $95 \% \mathrm{CI}$ \\
\hline True & 0.80036 & 0.80039 & $4.043 \cdot 10^{-5}$ & $(0.79946,0.80126)$ \\
Equal & 0.80036 & 0.80039 & $4.043 \cdot 10^{-5}$ & $(0.79946,0.80126)$ \\
Smaller & 0.81242 & 0.81253 & $19.38 \cdot 10^{-5}$ & $(0.81153,0.81331)$ \\
Bigger & 0.79781 & 0.79777 & $4.505 \cdot 10^{-5}$ & $(0.79691,0.79871)$ \\
\hline \hline$N=2000$ & Mean & Median & MSE & $95 \% \mathrm{CI}$ \\
\hline True & 0.80000 & 0.79993 & $7.635 \cdot 10^{-6}$ & $(0.79961,0.80039)$ \\
Equal & 0.80000 & 0.79993 & $7.635 \cdot 10^{-6}$ & $(0.79961,0.80039)$ \\
Smaller & 0.81194 & 0.81189 & $150.3 \cdot 10^{-6}$ & $(0.81154,0.81233)$ \\
Bigger & 0.79748 & 0.79740 & $13.91 \cdot 10^{-6}$ & $(0.79709,0.79787)$ \\
\hline \hline
\end{tabular}

Table 1 gives the mean squared error (MSE) and the 95\% confidence interval (95\% CI) for $b$. First, it is clear that when the weights satisfy the ratio condition (6), the parameter estimate of $b$ is closest to the true value of $b$ (i.e., it has the smallest MSE). If (6) does not hold, the resulting estimate of $b$ is biased. For Scenario 3 (Smaller), the parameter estimate has large positive bias; conversely, negative bias is observed for Scenario 4 (Bigger). When the regression's sample size is increased from $N=150$ to $N=2000$, the first two scenarios lead to a more accurate estimate of $b$. Although the MSEs for Scenarios 3 and 4 decrease with increasing sample size, these two scenarios still yield a parameter estimate of $b$ with significant bias (particularly for Scenario 3).

The (approximate) 95\% CI for $b$ is given in the last column of Table 1. For Scenarios 1 and 2, the 95\% CI always contains the true value $b=0.8$. For Scenarios 3 and 4, the confidence interval excludes the true value of $b=0.8$ for all three sample sizes. Figure 1 shows the boxplots of $\hat{b}_{l w s}$ under the four scenarios. A biased estimate of $b$ is observed for both Scenarios 3 and 4 , but 
the bias is clearly worse for Scenario 3 (Smaller). That is, when the ratio of the weights used is smaller than the correct ratio based on inverse variances, the bias is worse than if the ratio used were bigger.

We then tested the sensitivity of $\hat{b}_{l w s}$ to the ratio of regression weights using the relative inefficiency (RI), defined as the ratio of the MSE of $\hat{b}_{l w s}$ using a ratio of regression weights to that using the correct ratio, $\tilde{\sigma}_{y, i}^{2} / \tilde{\sigma}_{x, i}^{2}$. Then $\mathrm{RI} \geq 1$, and a misspecification of the ratio of regression weights corresponds to RI $>1$. The upper panel in Figure 2 shows how the RIs of $\hat{b}_{l w s}$ change with different values of the ratio of regression weights, $w_{x, i} / w_{y, i}$, where an RI as close to 1 as possible is preferred. We fixed $w_{x, i}$ at $1 / \tilde{\sigma}_{x, i}^{2}$ and varied the values of $w_{y, i}$ to obtain different ratios of regression weights. The correct ratio in this panel is $\tilde{\sigma}_{y, i}^{2} / \tilde{\sigma}_{x, i}^{2}=3$. It can be seen that the RIs of $\hat{b}_{l w s}$ are much more sensitive to Scenario 3 where the ratios have smaller values than the correct ratio of 3 , which corroborates our results in Table 1 and Figure 1.

The lower panel in Figure 2 shows the results for the case $\tilde{\sigma}_{y, i}^{2} / \tilde{\sigma}_{x, i}^{2}=1$. Notice that the correct ratio has decreased and, under Scenario 4 where the ratios have bigger values than the correct ratio of $\tilde{\sigma}_{y, i}^{2} / \tilde{\sigma}_{x, i}^{2}=1$, the RIs of $\hat{b}_{l w s}$ have worsened in comparison to the upper panel. Our general conclusion remains, that underestimation of $\operatorname{var}\left(Y_{i}\right) / \operatorname{var}\left(X_{i}\right)$ will lead to more severely biased estimates of $b$ than its overestimation.

We also did a simulation study for the model, $y_{i}=a+b x_{i}$, with both $a$ and $b$ to be estimated, which we call the nonzero-intercept case. We set the true regression relation to be, $y_{i}=1+0.8 x_{i}$, with other simulation settings the same as those in the zero-intercept case considered above (where recall that $\left.y_{i}=0.8 x_{i}\right)$. Similar results were obtained (not shown here) for the nonzerointercept case when estimating the slope $b$ : The "True" and "Equal" scenarios led to estimates of $b$ close to its true value. In contrast, the "Smaller" and "Bigger" scenarios led to estimates of $b$ with positive biases and negative biases, respectively. This mirrors the results we obtained for the zero-intercept case.

For the nonzero-intercept case, $y_{i}=a+b x_{i}$, the estimates of the intercept $a$ under the "True" and "Equal" scenarios are also close to its true value; the "Smaller" scenario leads to estimates of $a$ with negative biases (probably due to the overestimation of slope $b$ ), while the "Bigger" scenario leads to estimates of $a$ with positive biases (probably due to the underestimation of slope $b$ ). Therefore, to obtain consistent estimates of the regression coefficients, it is crucial to obtain consistent estimates of variances of the regression data $\left\{X_{i}, Y_{i}\right\}$ for defining the weights. In Sections 4 and 5 , we apply the preceding statistical methodology to the calibration of OCO-2 satellite data from TCCON ground-station data. 


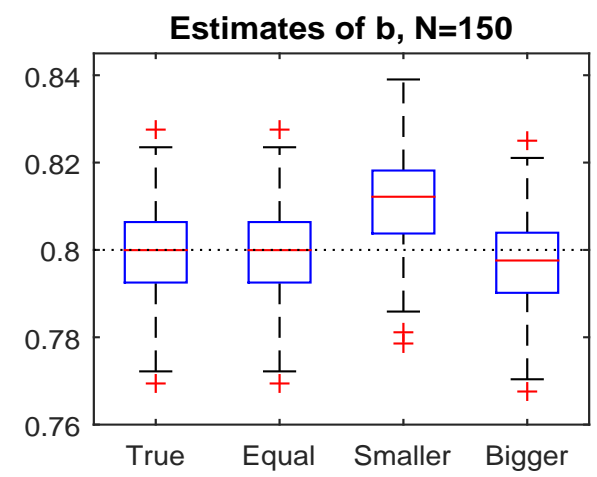

(a) $N=150$

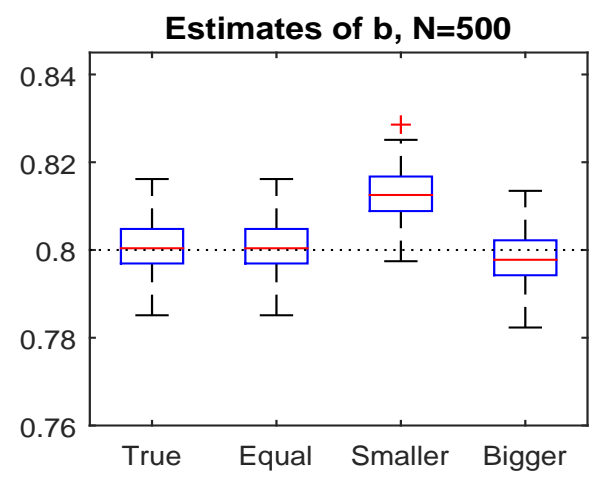

(b) $N=500$

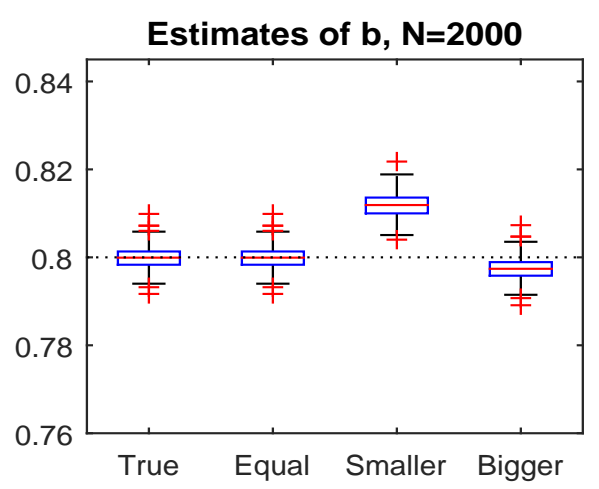

(c) $N=2000$

Fig. 1. Boxplots of the estimates of $b$ under different specifications of weights. The true value of $b$ is 0.8 .

\section{Statistical analysis of dependencies in the original TCCON and OCO-2 values}

Since both $X_{i}$ and $Y_{i}$ are aggregated data based on a dataset of individual TCCON observations and a dataset of individual OCO-2 observations, respectively, it is necessary to analyze the temporal (for TCCON) and spatial (for OCO-2) dependence structures in order to obtain variances of $X_{i}$ and $Y_{i}$. The TCCON and OCO-2 datasets at Lamont/3590 (which refers to the $i$-th station/orbit combination) are used to illustrate our methodology.

\subsection{TCCON data analysis}

There are more than 20 TCCON stations in the world and, in what follows, we have chosen the Lamont station located in Oklahoma, USA, to illustrate the appropriate calculation of $\tilde{\sigma}_{x, i}^{2}=\operatorname{var}\left(X_{i}\right)$ and $\tilde{\sigma}_{y, i}^{2}=\operatorname{var}\left(Y_{i}\right)$. The Lamont station is in the Southern Great Plains, which has been widely studied in climate-model-calibration contexts [25]. On orbit 3590, the OCO-2 satellite was in target mode, obtaining individual observations around the Lamont 


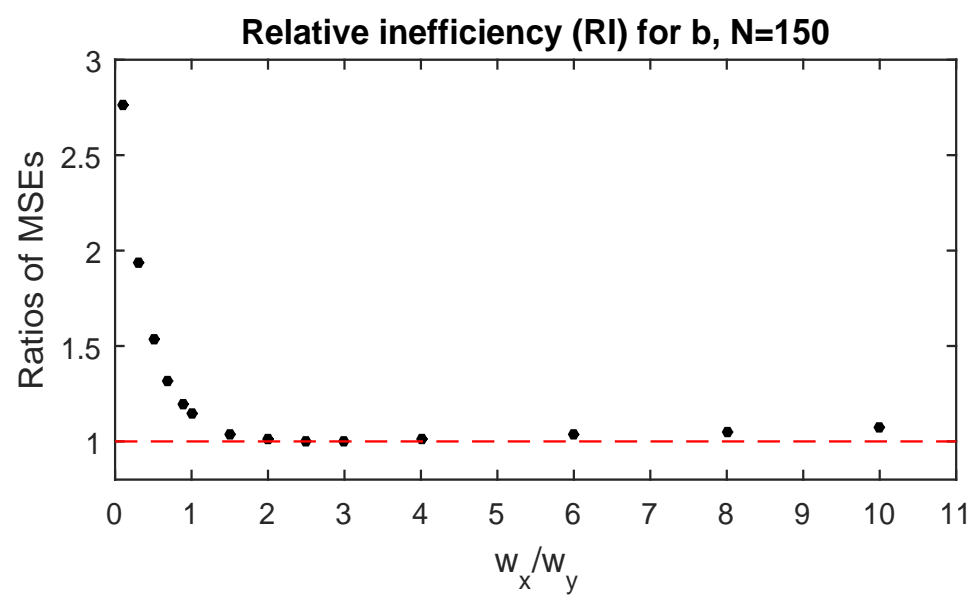

(a) $\tilde{\sigma}_{y, i}^{2}=1.5, \tilde{\sigma}_{x, i}^{2}=0.5\left(\tilde{\sigma}_{y, i}^{2} / \tilde{\sigma}_{x, i}^{2}=3\right)$.

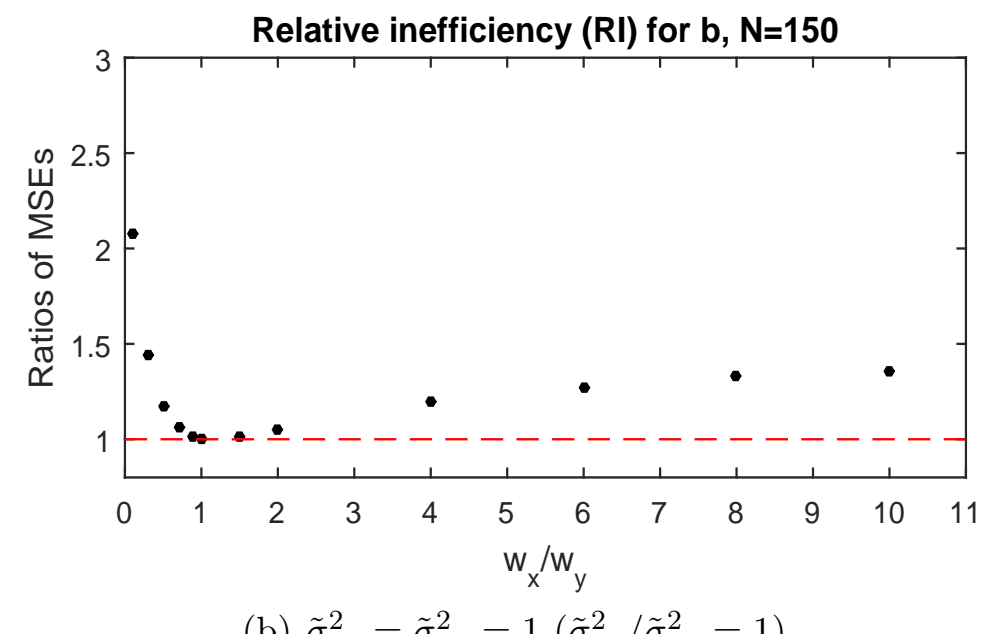

(b) $\tilde{\sigma}_{y, i}^{2}=\tilde{\sigma}_{x, i}^{2}=1\left(\tilde{\sigma}_{y, i}^{2} / \tilde{\sigma}_{x, i}^{2}=1\right)$.

Fig. 2. The relative inefficiency, or RI (defined as the ratio of MSEs), of $\hat{b}_{l w s}$ for different values of $w_{x, i} / w_{y, i}$. The dashed line at 1 shows the relative inefficiency of $\hat{b}_{l w s}$ using $w_{x, i} / w_{y, i}=\tilde{\sigma}_{y, i}^{2} / \tilde{\sigma}_{x, i}^{2}\left(\tilde{\sigma}_{y, i}^{2} / \tilde{\sigma}_{x, i}^{2}=3\right.$ for the upper panel, and $\tilde{\sigma}_{y, i}^{2} / \tilde{\sigma}_{x, i}^{2}=1$ for the lower panel). Large values of RI indicate an inferior estimator.

station during a time interval of a few minutes. In the analysis, the mean target time was first obtained as the average of OCO-2's target-start-time and target-end-time; then, as many as 65 individual TCCON observations, in the time window of approximately \pm 1 hour centered at the mean target time, were selected for statistical analysis. The left panel of Figure 3 shows the selected TCCON observations plotted against their observation times for Lamont/3590; the right panel shows the spatial locations of the OCO-2 observations from target-start-time to target-end-time.

Since TCCON observations at a given ground-monitoring station are taken over time, we model them as realizations from a temporal stochastic process (i.e., a time series). For the $i$-th station/orbit (here, Lamont/3590), let $\left\{X_{i, 1}, \ldots, X_{i, n_{x, i}}\right\}$ be the $n_{x, i}$ TCCON observations selected in the time win- 

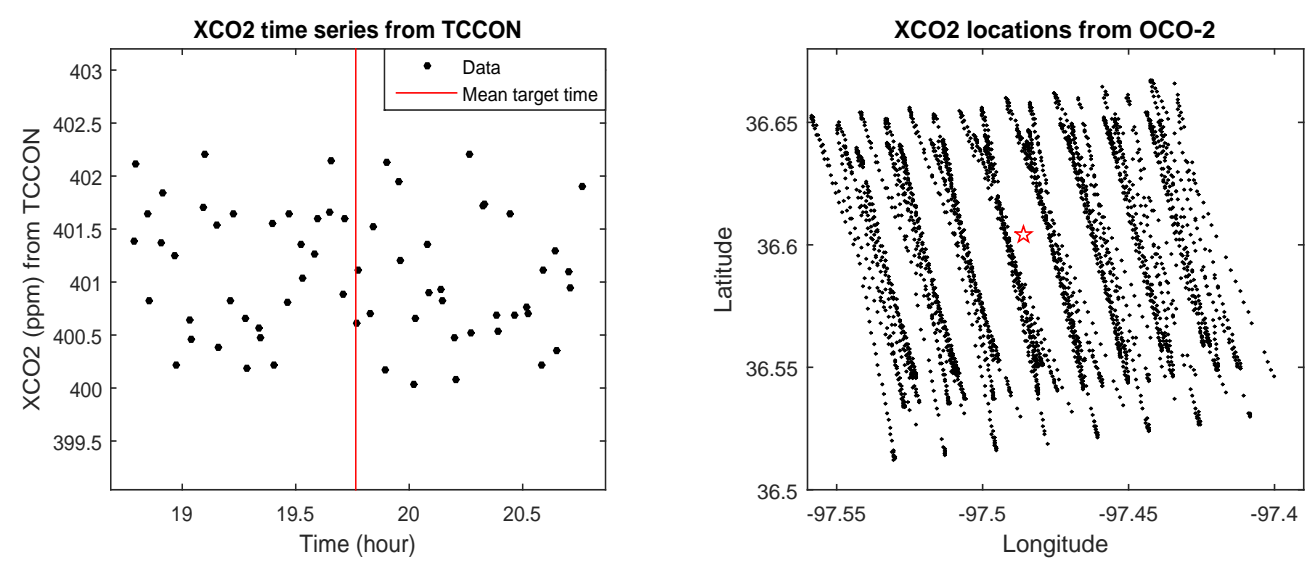

Fig. 3. The TCCON time series and OCO-2 observation locations for Lamont/3590. Left panel: TCCON observations versus time, where the vertical line is the mean target time. Right panel: OCO-2 observation locations, where the star shows the location of the Lamont TCCON station.

dow described above, and let $\left\{t_{i, 1}, \ldots, t_{i, n_{x, i}}\right\}$ be their corresponding observation times. We can generally expect $n_{x, i}=65$, although with missing data it may be less. We model $\left\{X_{i, j}\right\}_{j=1}^{n_{x, i}}$ as follows:

$$
X_{i, j}=x_{i}+\epsilon_{x, i, j},
$$

where $x_{i}$ is a fixed but unknown constant (in time) mean parameter, and $\epsilon_{x, i, j}$ is a measurement-error term that we assume to be Gaussian with mean zero and temporal covariance function, $\mathcal{C}_{x}\left(\cdot, \cdot ; \boldsymbol{\theta}_{x, i}\right)$. In what follows, we capture the temporal dependence through the exponential covariance function,

$$
\operatorname{cov}\left(\epsilon_{x, i, j}, \epsilon_{x, i, \ell}\right)=\mathcal{C}_{x}\left(t_{i, j}, t_{i, \ell} ; \boldsymbol{\theta}_{x, i}\right)=\sigma_{x, i}^{2} \exp \left(-\left|t_{i, j}-t_{i, \ell}\right| / \phi_{x, i}\right),
$$

where $\boldsymbol{\theta}_{x, i}=\left\{\sigma_{x, i}^{2}, \phi_{x, i}\right\}$, and $\sigma_{x, i}^{2}>0$ and $\phi_{x, i}>0$ are the variance and range parameters, respectively. In engineering applications of (8), $\phi_{x, i}$ is sometimes called the e-folding time, and $3 \phi_{x, i}$ is sometimes referred to as the equivalent range. For some TCCON stations/orbits, the time series might indicate a nonconstant trend over time. We remark that the covariance function can help capture small but apparent departures from a constant trend; if the trend component around the mean target time is not constant, then the range parameter increases to capture the temporal trend in the data.

By using REstricted Maximum Likelihood (REML) estimation for $\sigma_{x, i}^{2}$ and $\phi_{x, i}$ (see $\left.[26,27]\right)$, we obtain estimators of covariance parameters that are less biased than those obtained from maximum likelihood estimation. Let $P=$ $\left(I_{n_{x, i}}-\frac{1}{n_{x, i}} \mathbf{1}_{n_{x, i}} \mathbf{1}_{n_{x, i}}^{T}\right)$ be a projection matrix, where $\mathbf{1}_{n_{x, i}}$ is a column vector of $n_{x, i}$ ones; then REML performs maximum likelihood estimation on the 
transformed observations, $\tilde{\mathbf{X}}_{i}=P_{1:\left(n_{x, i}-1\right)} \mathbf{X}_{i}=P_{1:\left(n_{x, i}-1\right)} \boldsymbol{\epsilon}_{x, i}$, where $P_{1:\left(n_{x, i}-1\right)}$ is a sub-matrix of $P$ with its first $\left(n_{x, i}-1\right)$ rows, $\mathbf{X}_{i}=\left(X_{i, 1}, \ldots, X_{i, n_{x, i}}\right)^{T}$, and $\boldsymbol{\epsilon}_{x, i}=\left(\epsilon_{x, i, 1}, \ldots, \epsilon_{x, i, n_{x, i}}\right)^{T}$. Then $\hat{\boldsymbol{\theta}}_{x, i}$ is obtained by maximizing the following log-restricted-likelihood function:

$$
\ell\left(\boldsymbol{\theta}_{x, i} \mid\left\{X_{i, j}\right\}\right)=-\frac{1}{2} \tilde{\mathbf{X}}_{i}^{T} \tilde{\Sigma}_{x, i}^{-1} \tilde{\mathbf{X}}_{i}-\frac{1}{2} \log \left|\tilde{\Sigma}_{x, i}\right|-\frac{n_{x, i}}{2} \log (2 \pi),
$$

where $\tilde{\Sigma}_{x, i}=P_{1:\left(n_{x, i}-1\right)} \Sigma_{x, i} P_{1:\left(n_{x, i}-1\right)}^{T}$, and $\Sigma_{x, i}$ is the covariance matrix of $\mathbf{X}_{i}$. Notice that the $\log$-restricted-likelihood does not depend on the mean $x_{i}$.

The left panel in Figure 4 shows the empirical semivariogram (e.g., see [28]) and the fitted semivariogram using REML parameter estimates. The fitted semivariogram values match the empirical ones well. We can see that the fitted semivariogram reaches its sill quickly with increasing time lags, indicating very weak temporal dependence in the TCCON observations. Based on the estimates given in Section 5 , the equivalent range is $3 \hat{\phi}_{x, i} \simeq 1.3 \cdot 10^{-3}$ hour, which is much smaller than the time window of 2 hours.
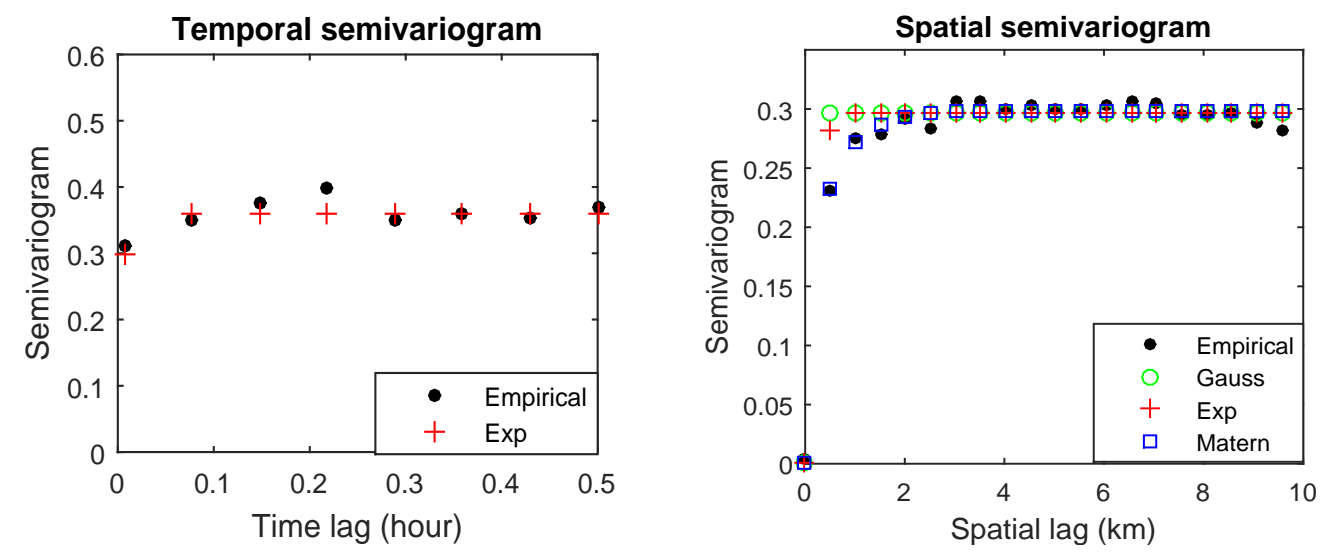

Fig. 4. Empirical and fitted semivariogram plots for Lamont/3590. Left panel: semivariograms for the TCCON (temporal semivariograms) observations. Right panel: semivariograms for the OCO-2 (spatial isotropic semivariograms) observations.

\subsection{OCO-2 data analysis}

The OCO-2 dataset that targets the TCCON ground station during a given orbit has spatio-temporal locations in a small spatial region $(\sim 0.2 \times 0.2$ degrees) within a few minutes ( $\sim 5$ minutes). Therefore, a spatial-constantmean assumption is likely to hold. Recall that the right panel in Figure 3 shows the locations of the OCO-2 data from target-start-time to target-end-time for Lamont/3590; eight footprints are clearly observed (and expected). 
Since the time interval of the OCO-2 observations is very short and the corresponding spatial locations are changing during the short time interval, we model the OCO-2 observations using a purely spatial process. Let $\left\{Y_{i, 1}, \ldots, Y_{i, n_{y, i}}\right\}$ be the $n_{y, i}$ OCO-2 observations for the $i$-th station/orbit, with corresponding spatial locations $\left\{\mathbf{s}_{i, 1}, \ldots, \mathbf{s}_{i, n_{y, i}}\right\}$. At the resolution of $15 \mathrm{~km}$, the chordal distance, which is a Euclidean distance in three-dimensional space, is appropriate to quantify "closeness" of the OCO-2 data locations.

We model $\left\{Y_{i, j}: j=1, \ldots, n_{y, i}\right\}$ as realizations from a spatial Gaussian process, as follows:

$$
Y_{i, j}=y_{i}+\epsilon_{y, i, j}
$$

where $y_{i}$ is a fixed but unknown spatially constant mean parameter, and $\epsilon_{y, i, j}$ is a measurement-error term that we assume to be Gaussian with mean zero and spatial covariance function, $\mathcal{C}_{y}\left(\cdot, \cdot ; \boldsymbol{\theta}_{y, i}\right)$. Let $\mathcal{C}_{y}\left(\cdot, \cdot ; \boldsymbol{\theta}_{y, i}\right)$ be the flexible isotropic Matérn covariance function (see $[28,29]$ ), which we use to evaluate $\operatorname{cov}\left(Y_{i, j}, Y_{i, \ell}\right)$. That is, the spatial covariances between OCO-2 data is modeled by

$$
\mathcal{C}_{y}\left(\mathbf{s}_{i, j}, \mathbf{s}_{i, \ell} ; \boldsymbol{\theta}_{y, i}\right)=\frac{\sigma_{y, i}^{2} 2^{1-\nu_{y, i}}}{\Gamma\left(\nu_{y, i}\right)}\left(\frac{\left\|\mathbf{s}_{i, j}-\mathbf{s}_{i, \ell}\right\|}{\phi_{y, i}}\right)^{\nu_{y, i}} \mathcal{K}_{\nu_{y, i}}\left(\frac{\left\|\mathbf{s}_{i, j}-\mathbf{s}_{i, \ell}\right\|}{\phi_{y, i}}\right),
$$

where $\boldsymbol{\theta}_{y, i}=\left\{\sigma_{y, i}^{2}, \phi_{y, i}, \nu_{y, i}\right\}, \sigma_{y, i}^{2}>0$ is the variance parameter, $\phi_{y, i}>0$ is the range parameter, and $\nu_{y, i}>0$ is the smoothness parameter. In $(10), \Gamma(\cdot)$ is the gamma function, and $\mathcal{K}_{\nu_{y, i}}(\cdot)$ is a modified Bessel function of the second kind of order $\nu_{y, i}$. The covariance model given by (10) provides extra flexibility for modeling the smoothness of the process with the inclusion of the third parameter, $\nu_{y, i}$. The exponential covariance function in (8) is a special case of the Matérn model with $\nu_{y, i}=0.5$; when $\nu_{y, i}$ tends to infinity, the so-called Gaussian covariance function is obtained.

Outliers are typical in most remote sensing datasets, and hence estimates of the covariance-model parameters need to be robust to them. We therefore use robust estimators of the semivariogram (e.g., $[28,30])$ and fit the covariancefunction parameters using weighted least squares [28].

The Cressie-Hawkins semivariogram estimator [28] is:

$$
\hat{\gamma}(\mathbf{h}(k))=\frac{1}{2}\left(\frac{1}{|N(\mathbf{h}(k))|} \sum_{N(\mathbf{h}(k))}\left|Y_{i, j}-Y_{i, \ell}\right|^{1 / 2}\right)^{4} /\left(0.457+\frac{0.494}{|N(\mathbf{h}(k))|}\right)
$$

where $N(\mathbf{h}(k)) \equiv\left\{(j, \ell): \mathbf{s}_{i, j}-\mathbf{s}_{i, \ell} \in \operatorname{tol}(\mathbf{h}(k))\right.$, and $\left.j, \ell=1, \ldots, n_{y, i}\right\}$, 
$\operatorname{tol}(\mathbf{h}(k))$ is a pre-specified tolerance region around the spatial lag $\mathbf{h}(k)$, and $|N(\mathbf{h}(k))|$ is the number of distinct pairs in $N(\mathbf{h}(k))$. Then the parameters $\boldsymbol{\theta}_{y, i}$ are estimated by weighted least squares (see [28]); that is, we minimize with respect to $\boldsymbol{\theta}_{y, i}$,

$$
W\left(\boldsymbol{\theta}_{y, i}\right)=\sum_{k=1}^{K}|N(\mathbf{h}(k))|\left(\frac{\hat{\gamma}(\mathbf{h}(k))}{\gamma\left(\mathbf{h}(k) ; \boldsymbol{\theta}_{y, i}\right)}-1\right)^{2} .
$$

In (12), $\gamma\left(\mathbf{h}(k) ; \boldsymbol{\theta}_{y, i}\right)=\mathcal{C}_{y}\left(\mathbf{0} ; \boldsymbol{\theta}_{y, i}\right)-\mathcal{C}_{y}\left(\mathbf{h}(k) ; \boldsymbol{\theta}_{y, i}\right)$ is the semivariogram based on the covariance model in (10). Notice that $\gamma\left(\cdot ; \boldsymbol{\theta}_{y, i}\right)$ does not depend on the spatial mean $y_{i}$, and hence neither does (12). The range of spatial lags $\{\mathbf{h}(k): k=1, \ldots, K\}$ is constrained so that $\|\mathbf{h}(k)\| \leq \frac{1}{2} \max \left\{\left\|\mathbf{s}_{i, j}-\mathbf{s}_{i, k}\right\|:\right.$ $\left.j, k=1, \ldots, n_{y, i}\right\}$, for each $k=1, \ldots, K$, and for each spatial lag we adjust $\operatorname{tol}(\mathbf{h}(k))$ to ensure that $|N(\mathbf{h}(k))|$ does not fall below 30; these are empirical rules of thumb that work well (e.g., see [31,32]). In our application to the Lamont/3590 OCO-2 dataset, $K=20$ equally spaced spatial lags were used to evaluate $W\left(\boldsymbol{\theta}_{y, i}\right)$ defined by $(12)$.

We fitted three Matérn models with different smoothness parameters, namely a Matérn model with $\hat{\nu}_{y, i}$ fitted via minimizing (12); the exponential model, $\sigma_{y, i}^{2} \exp \left(-\left\|\mathbf{s}_{i, j}-\mathbf{s}_{i, k}\right\| / \phi_{y, i}\right)$, which is a Matérn model with $\nu_{y, i}=0.5$; and the Gaussian model, $\sigma_{y, i}^{2} \exp \left(-\left\|\mathbf{s}_{i, j}-\mathbf{s}_{i, k}\right\|^{2} / \phi_{y, i}\right)$, which is a Matérn model with $\nu_{y, i} \rightarrow \infty$. The right panel of Figure 4 shows the empirical semivariograms versus the fitted semivariograms obtained from these three models. It is clear that the fitted Matérn covariance model is the best, due to its ability to capture the smoothness of the spatial process. The value of $W\left(\boldsymbol{\theta}_{y, i}\right)$ using the fitted Matérn model is 687.989, which is much smaller than the fitted exponential model's value of 1261.964 and much smaller than the fitted Gaussian model's value of 1384.135 .

The empirical semivariogram of the OCO-2 data attains its sill gently as a function of spatial lag, indicating quite strong spatial dependence. Based on the parameter-estimation results in Section 5, the equivalent range is about $1.37 \mathrm{~km}$. Compared with the spatial domain of approximately $15 \mathrm{~km} \times 15 \mathrm{~km}$, the correlations among the OCO-2 observations are substantial, and hence they will have a large effect on $\tilde{\sigma}_{y, i}^{2}=\operatorname{var}\left(Y_{i}\right)$, which will be in turn have a large effect on $\tilde{n}_{y, i}$, the effective sample size.

\section{Calculation of $X_{i}$ and $Y_{i}$ and of their associated variances}

In this section, we discuss how to estimate the variances of the measurement errors of $X_{i}$ in (7) and $Y_{i}$ in (9), which are then used to define the weights in 
(3). We have seen in Section 3 how important this step is; use of inappropriate values of $\tilde{\sigma}_{x, i}^{2}$ and $\tilde{\sigma}_{y, i}^{2}$ may lead to biased estimates of $b$. The TCCON and OCO-2 datasets at Lamont/3590 (indexed by $i$ ) [33] are used to illustrate the appropriate calculation of the all-important regression weights, $w_{x, i}=1 / \tilde{\sigma}_{x, i}^{2}$ and $w_{y, i}=1 / \tilde{\sigma}_{y, i}^{2}$, where asymptotically unbiased estimators, $\hat{\tilde{\sigma}}_{x, i}^{2}$ and $\hat{\tilde{\sigma}}_{y, i}^{2}$, are used in their place.

\subsection{Variance estimation in the TCCON dataset}

Generally speaking, TCCON datasets are of very high quality, with very few outliers. Consequently, the sample mean, $X_{i} \equiv \frac{1}{n_{x, i}} \sum_{j=1}^{n_{x, i}} X_{i, j}$, serves as the $i$-th representative point for TCCON in the regression analysis. From the model (7), $X_{i}=x_{i}+\operatorname{ave}\left(\epsilon_{x, i, j}\right)$, where ave $\left(\epsilon_{x, i, j}\right)=\frac{1}{n_{x, i}} \sum_{j=1}^{n_{x, i}} \epsilon_{x, i, j}$; hence,

$$
\tilde{\sigma}_{x, i}^{2}=\operatorname{var}\left(\operatorname{ave}\left(\epsilon_{x, i, j}\right)\right)=\frac{\sigma_{x, i}^{2}}{n_{x, i}}+\frac{1}{n_{x, i}^{2}} \sum_{j=1}^{n_{x, i}} \sum_{k \neq j} C_{x, i ; j, k},
$$

where $C_{x, i ; j, k}=\mathcal{C}_{x}\left(t_{i, j}, t_{i, k} ; \boldsymbol{\theta}_{x, i}\right)$ is given by (8). By substituting into (13) the REML estimates $\hat{\boldsymbol{\theta}}_{x, i}=\left\{\hat{\sigma}_{x, i}^{2}, \hat{\phi}_{x, i}\right\}$ (Section 4.1) of temporal covariance function parameters $\boldsymbol{\theta}_{x, i}$, the estimate $\hat{\tilde{\sigma}}_{x, i}^{2}$ of $\tilde{\sigma}_{x, i}^{2}$ can be readily obtained. Then we use the regression weight, $w_{x, i}=1 / \hat{\tilde{\sigma}}_{x, i}^{2}$.

If the measurement errors $\left\{\epsilon_{x, i, j}\right\}_{j=1}^{n_{x, i}}$ are independent, then $\operatorname{var}\left(X_{i}\right)=\sigma_{x, i}^{2} / n_{x, i}$. In the presence of temporal dependence, the effective sample size, $\tilde{n}_{x, i}$ (in contrast to the actual sample size), for computing the variance of $X_{i}$ is defined as (see [28], pp. 14-15):

$$
\tilde{n}_{x, i}=\sigma_{x, i}^{2} / \operatorname{var}\left(\operatorname{ave}\left(\epsilon_{x, i, j}\right)\right)=\left(\frac{1}{n_{x, i}^{2}} \sum_{j=1}^{n_{x, i}} \sum_{k=1}^{n_{x, i}} \rho_{x, i ; j, k}\right)^{-1}
$$

where $\rho_{x, i ; j, k}=C_{x, i ; j, k} / \sigma_{x, i}^{2}$. Formula (14) depends on $\sigma_{x, i}^{2}$ and $\phi_{x, i}$; estimates $\hat{\sigma}_{x, i}^{2}$ and $\hat{\phi}_{x, i}$ are substituted into (14) to obtain the final result that we also call $\tilde{n}_{x, i}$ (a slight abuse of notation). The effective sample size (14) is always smaller than the actual sample size when the measurement errors are positively correlated. For Lamont/3590, Table 2 shows that $\tilde{n}_{x, i}$ is very close to $n_{x, i}$, since there is only weakly positive temporal dependence in the TCCON dataset. 


\subsection{Variance estimation in the OCO-2 dataset}

OCO-2 measurements are based on reflected energy from Earth's surface. This, and the impact of environmental factors such as clouds and aerosols on the data-retrieval process, results in high variability and a number of outliers in the OCO-2 dataset. Hence, rather than the sample mean, the sample median given by $Y_{i}=\operatorname{median}\left\{Y_{i, j}: j=1, \ldots, n_{y, i}\right\} \equiv \operatorname{med}\left(Y_{i, j}\right)$ is chosen as a robust OCO-2 representative point in the pair $\left(X_{i}, Y_{i}\right)$ used in the regression. The OCO-2 data are spatially correlated, so we need to specify the (approximate) variance of the sample median under this dependence; detailed calculations are given in the Appendix.

From the model (9), $Y_{i}=y_{i}+\operatorname{med}\left(\epsilon_{y, i, j}\right)$, and hence $\tilde{\sigma}_{y, i}^{2}=\operatorname{var}\left(\operatorname{med}\left(\epsilon_{y, i, j}\right)\right)$. Under mild conditions that are given in [34,35], the large-sample variance is,

$$
\tilde{\sigma}_{y, i}^{2}=\operatorname{var}\left(\operatorname{med}\left(\epsilon_{y, i, j}\right)\right) \simeq \frac{\pi \sigma_{y, i}^{2}}{2 n_{y, i}}+\frac{\sigma_{y, i}^{2}}{n_{y, i}^{2}} \sum_{j=1}^{n_{y, i}} \sum_{k \neq j} \arcsin \left(C_{y, i ; j, k} / \sigma_{y, i}^{2}\right),
$$

where $C_{y, i ; j, k}=\operatorname{cov}\left(\epsilon_{y, i, j}, \epsilon_{y, i, k}\right)=\mathcal{C}_{y}\left(\mathbf{s}_{i, j}, \mathbf{s}_{i, k} ; \boldsymbol{\theta}_{y, i}\right)$ is given by (10). By substituting into (15) the semivariogram-based weighted-least-squares estimates, $\hat{\boldsymbol{\theta}}_{y, i}=\left\{\hat{\sigma}_{y, i}^{2}, \hat{\phi}_{y, i}, \hat{\nu}_{y, i}\right\}$ (Section 4.2), of spatial covariance parameters $\boldsymbol{\theta}_{y, i}$, the estimate $\hat{\tilde{\sigma}}_{y, i}^{2}$ of $\tilde{\sigma}_{y, i}^{2}$ can be readily obtained. Then we use the regression weight, $w_{y, i}=1 / \hat{\tilde{\sigma}}_{y, i}^{2}$.

If the measurement errors $\left\{\epsilon_{y, i, j}\right\}_{j=1}^{n_{y, i}}$ are independent, then $\operatorname{var}\left(Y_{i}\right) \simeq \frac{\pi}{2}\left(\sigma_{y, i}^{2} / n_{y, i}\right)$. Consequently, the effective sample size, $\tilde{n}_{y, i}$, for computing the variance of $Y_{i}$ is,

$$
\tilde{n}_{y, i}=\frac{\pi}{2} \cdot \frac{\sigma_{y, i}^{2}}{\operatorname{var}\left(\operatorname{med}\left(\epsilon_{y, i, j}\right)\right)}=\frac{\pi}{2}\left(\frac{1}{n_{y, i}^{2}} \sum_{j=1}^{n_{y, i}} \sum_{k=1}^{n_{y, i}} \arcsin \left(\rho_{y, i ; j, k}\right)\right)^{-1}
$$

where $\rho_{y, i ; j, k}=C_{y, i ; j, k} / \sigma_{y, i}^{2}$. Formula (16) depends on $\sigma_{y, i}^{2}, \phi_{y, i}$, and $\nu_{y, i}$; estimates $\hat{\sigma}_{y, i}^{2}, \hat{\phi}_{y, i}$, and $\hat{\nu}_{y, i}$ are substituted into (16) to obtain the final result that we also call $\tilde{n}_{y, i}$ (a slight abuse of notation). For Lamont/3590, Table 2 shows that the effective sample size $\tilde{n}_{y, i}$ is much smaller than the actual sample size $n_{y, i}$, since there is strongly positive spatial dependence in the OCO-2 dataset.

Similar variance calculations can be made for carrying out flux inversions, where the variances are needed for quantifying the uncertainty of spatially aggregated mole-fraction data. 


\subsection{Results for Lamont/3590}

Table 2 shows the results of parameter estimation and the calculation of effective sample sizes for both the TCCON and the OCO-2 datasets associated with Lamont/3590. For the TCCON dataset, the range-parameter estimate $\hat{\phi}_{x, i}$ is very small relative to the 2 -hour time window over which the data were collected, and so the temporal correlations drop quickly with increasing time lags. The weak correlations in the TCCON data lead to an effective sample size, $\tilde{n}_{x, i}$, that is very close to the actual sample size, $n_{x, i}$. For the OCO2 dataset, the range-parameter estimate $\hat{\phi}_{y, i}$ is much larger, relative to the $15 \mathrm{~km} \times 15 \mathrm{~km}$ spatial window in which the data were collected. Hence, the strongly positive spatial correlations in the OCO-2 data result in an effective sample size, $\tilde{n}_{y, i}$, that is much smaller than the actual sample size, $n_{y, i}$.

Table 2

Parameter estimation results, sample variances, and the effective sample sizes for Lamont/3590.

\begin{tabular}{|c|ccc|cc|ccc|}
\hline \hline TCCON & $X_{i}$ & $\hat{\sigma}_{x, i}^{2}$ & $S_{x, i}^{2}$ & $n_{x, i}$ & $\tilde{n}_{x, i}$ & $\hat{\sigma}_{x, i}^{2}$ & $\hat{\phi}_{x, i}$ & $\hat{\nu}_{x, i}$ \\
\hline & 401.0840 & 0.0063 & 0.3602 & 65 & 57.05 & 0.3607 & 0.0039 & $0.5($ fixed $)$ \\
\hline OCO-2 & $Y_{i}$ & $\hat{\tilde{\sigma}}_{y, i}^{2}$ & $S_{y, i}^{2}$ & $n_{y, i}$ & $\tilde{n}_{y, i}$ & $\hat{\sigma}_{y, i}^{2}$ & $\hat{\phi}_{y, i}$ & $\hat{\nu}_{y, i}$ \\
\hline & 400.0395 & 0.0023 & 0.3025 & 2961 & 202.32 & 0.2989 & 0.7117 & 0.1849 \\
\hline \hline
\end{tabular}

Based on (6), we obtain the relative regression weights of $w_{x, i} / w_{y, i}=\tilde{\sigma}_{y, i}^{2} / \tilde{\sigma}_{x, i}^{2}=$ 0.3651. For version 7 of the OCO-2 retrieval data product, the calculation used $w_{x, i} / w_{y, i}=S_{y, i}^{2} / S_{x, i}^{2}=0.8398$, where $S_{x, i}^{2}$ and $S_{y, i}^{2}$ are sample variances of the individual TCCON and OCO-2 datasets, respectively. The sample variances were used to define weights for version 7 of the OCO-2 retrieval data product, because they better reflect variability observed in the data than the squared standard errors. Based on our calculations in this article, the sample-variancebased weights used for version 7 overestimate the approximate ratio of weights for Lamont/3590 (i.e., Scenario 4, which is "Bigger"). Our simulations in Section 3 show that the regression-slope estimate will be negatively biased and the relative inefficiency will be larger than 1, but not as high as one would obtain under Scenario 3 ("Smaller").

\section{Concluding remarks}

In this paper, we have proposed a statistical procedure for obtaining regression weights that lead to consistent estimation of linear-regression coefficients. Our application is to calibration of satellite remote sensing observa- 
tions obtained by looking down from space, calibrated to ground-based observations obtained by looking up from the ground. Specifically, the OCO-2 values $\left\{Y_{i}\right\}$ are regressed on the corresponding TCCON values $\left\{X_{i}\right\}$. In this article, we show that the appropriate regression weights depend on temporal (TCCON) and spatial (OCO-2) dependence structures. Specification of the regression weights associated with $X_{i}$ and $Y_{i}$ are crucial for obtaining an (asymptotically) unbiased, least-sum-of-weighted-squares estimator, $\hat{b}_{l w s}$. When $w_{x, i} / w_{y, i}=\tilde{\sigma}_{y, i}^{2} / \tilde{\sigma}_{x, i}^{2}$, the estimating equation for $b$ is unbiased, which results in (asymptotic) unbiasedness of $\hat{b}_{l w s}$. Therefore, it is desirable to use unbiased estimates of variances of $X_{i}$ and $Y_{i}$ for defining the weights.

Since $X_{i}$ and $Y_{i}$ are aggregated data calculated from sets of individual TCCON and OCO-2 observations, respectively, we explore the temporal-dependence and spatial-dependence structures in the TCCON and OCO-2 datasets for estimating $\tilde{\sigma}_{x, i}^{2}$ and $\tilde{\sigma}_{y, i}^{2}$, respectively. Based on our analysis, the individual observations in the TCCON dataset are weakly correlated in time, resulting in an effective sample size very close to the actual sample size; in contrast, the individual observations in the OCO-2 dataset have nonnegligible correlations in space, resulting in an effective sample size much smaller than the actual sample size. Our results show that any new version of the OCO-2 data obtained by regressing OCO-2 $(Y)$ on TCCON $(X)$ should use regression weights that result in unbiased estimating equations of the regression parameters.

NASA's latest release of OCO-2 data is version 7; it was obtained from a linearregression fit according to the model given by (1), with intercept $a=0$ and a different choice of regression weights than the optimal choice proposed in this paper; see Section 5. Version 7 used TCCON and their concomitant OCO-2 datasets at $N=66$ station/orbit combinations. Future work will result in a careful analysis of all 66 datasets, which can then be used to obtain consistent estimators, $\hat{a}_{l w s}$ and $\hat{b}_{l w s}$, of the regression parameters $a$ and $b$. The research will build on the substantial methodology given in the preceding sections and the appendix.

\section{Acknowledgements}

The OCO-2 data used in this article were produced by the OCO-2 project at the Jet Propulsion Laboratory, California Institute of Technology, and they were obtained from the OCO-2 data archive maintained at the NASA Goddard Earth Science Data and Information Services Center (http://disc.sci . gsfc.nasa.gov/OCO-2). TCCON data were obtained from the TCCON Data Archive, hosted by the Carbon Dioxide Information Analysis Center (CDIAC) (tccon.ornl.gov). The Lamont TCCON station is funded by NASA grants NNX14AI60G, NNX11AG01G, NAG5-12247, NNG05-GD07G, and NASA's Orbiting Carbon Observatory Program. We are grateful to the DOE ARM program for technical support at Lamont. Zhang and Cressie's research was 
partially supported by a 2015-2017 Australian Research Council Discovery Grant, number DP150104576; Cressie's research was also partially supported by NASA grant NNH11-ZDA001N-OCO2. We would like to thank Paul Wennberg and Camille Viatte for early discussions and their interest in this research.

\section{References}

[1] D. Crisp, H. R. Pollock, R. Rosenberg, L. Chapsky, R. A. M. Lee, F. A. Oyafuso, et al., The on-orbit performance of the Orbiting Carbon Observatory (OCO-2) instrument and its radiometrically calibrated products, Atmospheric Measurement Techniques Discussions (2016).

[2] R. K. Pachauri, M. Allen, V. Barros, J. Broome, W. Cramer, R. Christ, J. Church, L. Clarke, Q. Dahe, P. Dasgupta, et al., Climate Change 2014: Synthesis Report. Contribution of Working Groups i, ii and iii to the Fifth Assessment Report of the Intergovernmental Panel on Climate Change, Tech. rep. (2014).

[3] C. L. Quéré, R. J. Andres, T. Boden, T. Conway, R. Houghton, J. I. House, G. Marland, G. P. Peters, G. Van der Werf, A. Ahlström, et al., The global carbon budget 1959-2011, Earth System Science Data 5 (2013) 165-185.

[4] C. Le Quéré, M. R. Raupach, J. G. Canadell, G. Marland, L. Bopp, P. Ciais, T. J. Conway, S. C. Doney, R. A. Feely, P. Foster, et al., Trends in the sources and sinks of carbon dioxide, Nature Geoscience 2 (2009) 831-836.

[5] S. Boland, H. Bösch, L. Brown, J. Burrows, P. Ciais, B. Connor, D. Crisp, S. Denning, S. Doney, R. Engelen, et al., The need for atmospheric carbon dioxide measurements from space: Contributions from a rapid reflight of the Orbiting Carbon Observatory, Tech. rep., OCO Science Team, NASA Jet Propulsion Labaratory (2009).

[6] C. Miller, D. Crisp, P. DeCola, S. Olsen, J. T. Randerson, A. M. Michalak, A. Alkhaled, P. Rayner, D. J. Jacob, P. Suntharalingam, et al., Precision requirements for space-based data, Journal of Geophysical Research: Atmospheres 112 (D10) (2007) n/a-n/a. doi:10.1029/2006JD007659.

[7] D. Wunch, G. C. Toon, J.-F. L. Blavier, R. A. Washenfelder, J. Notholt, B. J. Connor, D. W. Griffith, V. Sherlock, P. O. Wennberg, The total carbon column observing network, Philosophical Transactions of the Royal Society of London A: Mathematical, Physical and Engineering Sciences 369 (2011) 2087-2112.

[8] D. Wunch, G. C. Toon, P. O. Wennberg, S. C. Wofsy, B. B. Stephens, M. L. Fischer, O. Uchino, J. B. Abshire, P. Bernath, S. C. Biraud, et al., Calibration of the Total Carbon Column Observing Network using aircraft profile data, Atmospheric Measurement Techniques 3 (2010) 1351-1362. 
[9] D. Wunch, P. O. Wennberg, G. Osterman, B. Fisher, B. Naylor, C. M. Roehl, C. O'Dell, et al., Comparisons of the Orbiting Carbon Observatory-2 (OCO2) XCO2 measurements with TCCON, Atmospheric Measurement Techniques Discussions (2016).

[10] L. Mandrake, C. O'Dell, D. Wunch, P. O. Wennberg, B. Fisher, G. B. Osterman, A. Eldering, Orbiting Carbon Observatory-2 (OCO-2) warn level, bias correction, and lite file product description, Tech. rep., JPL (2015).

[11] C. W. O’Dell, P. O. Wennberg, A. Eldering, D. Crisp, M. R. Gunson, B. Fisher, The OCO-2 retrieval algorithm, Atmospheric Measurement Techniques, in prep. (2016).

[12] J. Wennberg, A. Gittelsohn, Small area variations in health care delivery, Science 182 (1973) 1102-1108.

[13] Goddard Earth Sciences Data and Information Services Center OCO-2 Data Holdings (2016). doi:http://disc.sci.gsfc.nasa.gov/OCO-2.

[14] D. York, Least-squares fitting of a straight line, Canadian Journal of Physics 44 (1966) 1079-1086.

[15] D. York, N. M. Evensen, M. L. Martınez, J. D. B. Delgado, Unified equations for the slope, intercept, and standard errors of the best straight line, American Journal of Physics 72 (2004) 367-375.

[16] J. Riu, F. X. Rius, Univariate regression models with errors in both axes, Journal of Chemometrics 9 (1995) 343-362.

[17] J. Riu, F. X. Rius, Assessing the accuracy of analytical methods using linear regression with errors in both axes, Analytical Chemistry 68 (1996) 1851-1857.

[18] C. Cantrell, Technical Note: Review of methods for linear least-squares fitting of data and application to atmospheric chemistry problems, Atmospheric Chemistry and Physics 8 (2008) 5477-5487.

[19] W. A. Fuller, Measurement Error Models, John Wiley \& Sons, Hoboken, NJ, 2009.

[20] C.-L. Cheng, J. Riu, On estimating linear relationships when both variables are subject to heteroscedastic measurement errors, Technometrics 48 (2006) $511-519$.

[21] D. York, Least squares fitting of a straight line with correlated errors, Earth and Planetary Science Letters 5 (1968) 320-324.

[22] D. M. Titterington, A. N. Halliday, On the fitting of parallel isochrons and the method of maximum likelihood, Chemical Geology 26 (1979) 183-195.

[23] V. P. Godambe, An optimum property of regular maximum likelihood estimation, Annals of Mathematical Statistics 31 (1960) 1208-1211.

[24] G. Y. Yi, N. Reid, A note on mis-specified estimating functions, Statistica Sinica 20 (2010) 1749-1769. 
[25] B. Yang, Y. Qian, G. Lin, R. Leung, Y. Zhang, Some issues in uncertainty quantification and parameter tuning: A case study of convective parameterization scheme in the WRF regional climate model, Atmospheric Chemistry and Physics 12 (2012) 2409-2427.

[26] H. D. Patterson, R. Thompson, Recovery of inter-block information when block sizes are unequal, Biometrika 58 (1971) 545-554.

[27] D. A. Harville, Maximum likelihood approaches to variance component estimation and to related problems, Journal of the American Statistical Association 72 (1977) 320-338.

[28] N. Cressie, Statistics for Spatial Data, revised edition, John Wiley \& Sons, Hoboken, NJ, 1993.

[29] B. Matérn, Spatial Variation, Vol. 36, Springer Science \& Business Media, New York, NY, 2013.

[30] N. Cressie, D. M. Hawkins, Robust estimation of the variogram: I, Journal of the International Association for Mathematical Geology 12 (1980) 115-125.

[31] A. G. Journel, C. J. Huijbregts, Mining Geostatistics, Academic Press, London, UK, 1978.

[32] N. Cressie, A graphical procedure for determining nonstationarity in time series, Journal of the American Statistical Association 83 (1988) 1108-1116.

[33] P. O. Wennberg, D. Wunch, C. Roehl, J.-F. Blavier, G. C. Toon, N. Allen, P. Dowell, K. Teske, C. Martin, J. Martin, TCCON data from Lamont, Oklahoma, USA (2014). doi:10.14291/tccon.ggg2014.lamont01.R0/1149159.

[34] N. Cressie, G. Glonek, Median based covariogram estimators reduce bias, Statistics \& Probability Letters 2 (1984) 299-304.

[35] P. K. Sen, On the Bahadur representation of sample quantiles for sequences of $\varphi$-mixing random variables, Journal of Multivariate Analysis 2 (1972) 77-95.

[36] I. Abrahamson, Orthant probabilities for the quadrivariate normal distribution, Annals of Mathematical Statistics 35 (1964) 1685-1703.

\section{APPENDIX . Approximate variance of the sample median under dependence}

We provide some details for obtaining the approximate variance of the sample median under dependence. Since many of the target-mode OCO-2 datasets contain outliers, the sample median replaces the sample mean as the representative value, $Y_{i}$, for the $i$-th station/orbit. Recall that we model the $i$-th OCO-2 dataset as a realization from a Gaussian process with a constant mean $y_{i}$ and a spatial covariance function, $\mathcal{C}_{y}\left(\cdot, \cdot ; \boldsymbol{\theta}_{y, i}\right)$ given by (10). Under mild 
conditions, the sample median converges almost surely to $y_{i}$ and, to leading order, we may write the sample median as (see $[34,35]$ ),

$$
\tilde{Y}_{i}=y_{i}+\frac{1}{n_{y, i}} \sum_{j=1}^{n_{y, i}} \operatorname{sgn}\left(Y_{i, j}-y_{i}\right) /\left(2 f\left(y_{i}\right)\right) .
$$

In the equation above, the function, $\operatorname{sgn}(x)$, is a sign function such that $\operatorname{sgn}(x)=1$ if $x>0, \operatorname{sgn}(x)=0$ if $x=0$, and $\operatorname{sgn}(x)=-1$ if $x<0$; and $f(\cdot)$ is the density function of $Y_{i, j}$, which here is Gaussian.

Therefore, the asymptotic variance of the sample median is, to leading order,

$$
\begin{aligned}
\operatorname{var}\left(\tilde{Y}_{i}\right) & =\frac{1}{n_{y, i}^{2}} \sum_{j=1}^{n_{y, i}} \sum_{k=1}^{n_{y, i}} \operatorname{cov}\left(\operatorname{sgn}\left(Y_{i, j}-y_{i}\right), \operatorname{sgn}\left(Y_{i, k}-y_{i}\right)\right) /\left(2 f\left(y_{i}\right)\right)^{2} \\
& =\frac{1}{n_{y, i}^{2}} \sum_{j=1}^{n_{y, i}} \sum_{k=1}^{n_{y, i}} E\left(\operatorname{sgn}\left(Y_{i, j}-y_{i}\right) \operatorname{sgn}\left(Y_{i, k}-y_{i}\right)\right) /\left(2 f\left(y_{i}\right)\right)^{2} \\
& =\frac{1}{n_{y, i}^{2}} \sum_{j=1}^{n_{y, i}} \frac{1}{\left(2 f\left(y_{i}\right)\right)^{2}}+\frac{1}{n_{y, i}^{2}} \sum_{j=1}^{n_{y, i}} \sum_{k \neq j} E\left(\operatorname{sgn}\left(Y_{i, j}-y_{i}\right) \operatorname{sgn}\left(Y_{i, k}-y_{i}\right)\right) /\left(2 f\left(y_{i}\right)\right)^{2},
\end{aligned}
$$

since $E\left(\operatorname{sgn}\left(Y_{i, j}\right)\right)=0$ and $E\left(\operatorname{sgn}\left(Y_{i, j}\right)^{2}\right)=1$. Now,

$$
\begin{aligned}
E\left(\operatorname{sgn}\left(Y_{i, j}-y_{i}\right) \operatorname{sgn}\left(Y_{i, k}-y_{i}\right)\right)= & E\left(\mathbf{1}_{Y_{i, j}>y_{i}} \mathbf{1}_{Y_{i, k}>y_{i}}\right)-E\left(\mathbf{1}_{Y_{i, j}>y_{i}} \mathbf{1}_{Y_{i, k}<y_{i}}\right) \\
& -E\left(\mathbf{1}_{Y_{i, j}<y_{i}} \mathbf{1}_{Y_{i, k}>y_{i}}\right)+E\left(\mathbf{1}_{Y_{i, j}<y_{i}} \mathbf{1}_{Y_{i, k}<y_{i}}\right) \\
= & P\left(Y_{i, j}>y_{i}, Y_{i, k}>y_{i}\right)-P\left(Y_{i, j}>y_{i}, Y_{i, k}<y_{i}\right) \\
& -P\left(Y_{i, j}<y_{i}, Y_{i, k}>y_{i}\right)+P\left(Y_{i, j}<y_{i}, Y_{i, k}<y_{i}\right) .
\end{aligned}
$$

By Sheppard's theorem described in [36], $P\left(Y_{i, j}>y_{i}, Y_{i, k}>y_{i}\right)=\frac{1}{4}+$ $\frac{1}{2 \pi} \arcsin \left(C_{y, i ; j, k} /\left(C_{y, i ; j, j} C_{y, i ; k, k}\right)^{1 / 2}\right)$, where $C_{y, i ; j, k}$ is the covariance of $Y_{i, j}$ and $Y_{i, k}$ and, because of stationarity of (10), $C_{y, i ; j, j}=C_{y, i ; k, k}=\sigma_{y, i}^{2}$. Since $P\left(Y_{i, j}<\right.$ $\left.y_{i}, Y_{i, k}<y_{i}\right)=P\left(Y_{i, j}>y_{i}, Y_{i, k}>y_{i}\right)$, and

$$
P\left(Y_{i, j}>y_{i}, Y_{i, k}<y_{i}\right)+P\left(Y_{i, j}<y_{i}, Y_{i, k}>y_{i}\right)=1-2 P\left(Y_{i, j}>y_{i}, Y_{i, k}>y_{i}\right),
$$

we readily obtain,

$$
E\left(\operatorname{sgn}\left(Y_{i, j}-y_{i}\right) \operatorname{sgn}\left(Y_{i, k}-y_{i}\right)\right)=\frac{2}{\pi} \arcsin \left(C_{y, i ; j, k} /\left(C_{y, i ; j, j} C_{y, i ; k, k}\right)^{1 / 2}\right) .
$$

Recall that in the Gaussian case, the marginal density results in $f\left(y_{i}\right)=$ $\left(2 \pi \sigma_{y, i}^{2}\right)^{-1 / 2}$, and hence $1 /\left(2 f\left(y_{i}\right)\right)^{2}=\pi \sigma_{y, i}^{2} / 2$. Therefore, the asymptotic variance of the sample median is, 


$$
\begin{aligned}
\operatorname{var}\left(\operatorname{med}\left(Y_{i, j}\right)\right) & =\frac{1}{n_{y, i}} \cdot \frac{\pi \sigma_{y, i}^{2}}{2}+\frac{1}{n_{y, i}^{2}} \sum_{j=1}^{n_{y, i}} \sum_{k \neq j} \frac{2}{\pi} \arcsin \left(C_{y, i ; j, k} / \sigma_{y, i}^{2}\right) \cdot \frac{\pi \sigma_{y, i}^{2}}{2} \\
& =\frac{\sigma_{y, i}^{2}}{n_{y, i}}\left(\frac{\pi}{2}\right)+\frac{\sigma_{y, i}^{2}}{n_{y, i}^{2}} \sum_{y=1}^{2} \sum_{k \neq j} \arcsin \left(\rho_{y, i ; j, k}\right) \\
& =\frac{\sigma_{y, i}^{2}}{n_{y, i}^{2}} \sum_{j=1}^{n_{y, i}} \sum_{k=1}^{n_{y, i}} \arcsin \left(\rho_{y, i ; j, k}\right),
\end{aligned}
$$

where $\rho_{y, i ; j, k} \equiv C_{y, i ; j, k} / \sigma_{y, i}^{2}$ is the spatial correlation between $Y_{i, j}$ and $Y_{i, k}$. 